\title{
What Has Mathematics Done for Biology?
}

\author{
Michael C. Mackey • Philip K. Maini
}

Published online: 16 May 2015

(C) Society for Mathematical Biology 2015

\begin{abstract}
"What has mathematics done for biology?" is a question that every mathematical biologist has been asked at some time, or has asked themselves. While bioinformatics has been very successful and widely accepted in biology, the acceptance of mathematical biology has been slower. Of course, there are notable exceptions-in ecology and epidemiology there is a long history of mathematical modelling [see, e.g., the books by Murray (2002, 2003) and May and McLean (2007)], in physiology the HodgkinHuxley model is well known [see, e.g., the book by Keener and Sneyd (2009)], while in pattern formation the Turing model (Turing 1952), while still controversial, has certainly stimulated an enormous amount of experimental activity and led to the paradigm patterning principle of short-range activation, long-range inhibition (Gierer and Meinhardt 1972).
\end{abstract}

These successes for mathematical biology are significant, but still relatively rare, bearing in mind that the field has matured and grown substantially over the past 50 years. In fact, during the past 20 years bioinformatics has come into being and taken over mathematical biology in the race to the laboratory. There are a number of reasons for this: (1) there have been few major problems that mathematical biology has successfully addressed (compared with the genome project), (2) for validation, models in mathematical biology typically rely on spatiotemporally resolved data and, to date, data tend to be static (electrophysiology is an exception) and therefore more amenable to the tools of bioinformatics. However, mathematical biology has made numerous advances in biology, and advances in technology (particularly imaging) are leading to

\footnotetext{
M. C. Mackey $(\varangle)$

Montreal, QC, Canada

e-mail: michael.mackey@mcgill.ca

P. K. Maini

Oxford, UK

e-mail:maini@maths.ox.ac.uk
} 
the generation of data that are appropriate for dynamic models. In this special issue, we present a number of examples of successes in mathematical biology.

Dale and Mackey (2014) discuss their work over a 40+ year period in trying to understand the origins of the periodic haematological disease cyclical neutropenia (CN). Through a combination of mathematical modelling and analysis of laboratory and clinical data, it is now probable that $\mathrm{CN}$ is due to a Hopf bifurcation in the dynamics of the haematological stem cells that leads to oscillations and that this bifurcation can be traced to abnormally high levels of apoptosis in proliferating neutrophil precursors. The experimental/clinical and modelling work also led to the successful use of a recombinant cytokine in the treatment of this disease. They finally show how these insights can be used to better administer chemotherapy to avoid some of the side effects.

Friedman and Hao (2014) consider the problem of plaque formation in atherosclerosis. They describe a mechanistic model which allowed them to define a "risk map" for the disease depending on the levels of LDL and HDL, and their predictions agree with the general guidelines of the American Heart Association (AHA). The modelling allows exploration of plaque regression under different drug treatments. The results qualitatively agree with experiments in mice and suggest possible avenues for drug treatments in humans.

Glass and Shrier (2014) look back at the development of theory for excitable media, beginning with Hodgkin-Huxley. They raise the important point that there are many different ways to model the same process, ranging from very biologically detailed and computationally expensive models, to phenomenological models which aim to capture the essence of the process in a very simple way. While the former approach allows one to include a lot of biological detail and aims to predict the effect on tissuelevel behaviour of precise intracellular manipulation, the latter allows for more "highlevel" predictions. Focussing in particular on the latter, they show how simple models have led to understanding how aggregates of heart cells would respond to different types of electrical stimulation and how waves propagate across cardiac tissue, and the identification of the types of instabilities that may be the cause of often fatal arrhythmias, such as ventricular tachycardia and ventricular fibrillation.

Tyson and Novak (2014) show how mathematical modelling can be used to address the outcome of complex molecular regulatory networks. They focus on the dynamics of the CDK network which controls progress through the eukaryotic cell cycle. This complicated system involves many interacting feedback loops whose collective dynamics cannot be understood by verbal reasoning alone. In a large body of highly significant work, they analysed the outcome of these interactions using a dynamical systems approach and made a number of predictions which were verified experimentally.

Umulis and Othmer (2014) address a number of aspects of the problem of pattern formation in development, including robustness and scale invariance, before focussing on the paradigm model of the fruit fly Drosophila. The signalling and regulation during patterning in the early embryonic phase is viewed as a sequence of interacting modules which are integrated to provide a full spatiotemporal model consisting of coupled nonlinear partial differential equations. In the wing disc, patterning and growth are intimately linked, and understanding how growth is controlled requires models that 
link individual cell behaviour to tissue-level control. Throughout this paper, it is shown how experiment and theory inform each other.

Jackson at al. (2015) tackle the field of mathematical neuro-oncology with the particular example of glioblastoma. They show how a minimal model of cell proliferation and movement can capture many of the key features of the spread of this tumour. They further show how this simple model has been parameterised through patient data and used to derive patient-specific tumour properties that allow prediction of the effects of different therapies.

Mangel (2014) reviews how stochastic dynamic programming (SDP) allows us to study evolution by relating the physiological state of an organism with its environment. Two canonical equations for SDP are derived and applied to insect behavioural ecology, developmental pathways in steelhead trout, and skipped spawning in Norwegian cod. Again, model development and prediction go hand in hand with empirical data collection.

Bioinformatics and mathematical biology can be thought of as complementary to each other. To draw an analogy, Kepler was one of the early "big data" analysts who came up with the theory of planetary motion. Had we decided there and then the problem of how the planets orbit around the sun had been understood, could we have used these insights to eventually land people on the moon? Newton, by providing the mechanism, developed the theory underlying these data-driven laws which allowed us a full understanding of planetary motion.

The small sample of papers in this special issue illustrates a number of important aspects of mathematical biology. Firstly, they show the very wide range of mathematical techniques that have been used (ordinary, partial, stochastic, delay differential equations). Secondly, to make impact in the field and discover new biology, the theoretician must be application driven, rather than technique driven. Thirdly, the search for new biology invariably leads to new mathematics. Finally, to be taken seriously by biologists, there must be significant interaction with experimentalists both in terms of model development and the testing of model-derived predictions.

As John Bonner said long ago (Othmer et al. 2009), commenting specifically on developmental biology, "We have arrived at the stage where models are useful to suggest experiments, and the facts of the experiments in turn lead to new and improved models that suggest new experiments. By this rocking back and forth between the reality of experimental facts and the dream world of hypotheses, we can move slowly toward a satisfactory solution of the major problems of developmental biology." This special issue presents papers illustrating this "rocking back and forth" to varying degrees in a number of application areas. It shows that mathematical biology has had an important impact in biology and, as quantification in biology continues apace, we are at the dawning of a new era in which mathematical biology will increasingly gain acceptance in biology and impact the subject on a scale not seen to date.

\section{References}

Dale DC, Mackey MC (2014) Understanding, treating and avoiding hematological disease: better medicine through mathematics? Bull Math Biol. doi:10.1007/s11538-014-9995-X 
Friedman A, Hao W (2014) A mathematical model of atherosclerosis with reverse cholesterol transport and associated risk factors. Bull Math Biol. doi:10.1007/s11538-014-0010-3

Gierer A, Meinhardt H (1972) A theory of biological pattern formation. Kybernetik 12:30-39

Glass L, Shrier A (2014) Functional characterization of oscillatory and excitable media. Bull Math Biol. doi:10.1007/s11538-014-0015-y

Jackson PR, Juliano J, Hawkins-Daarud A, Rockne RC, Swanson K (2015) Patient specific mathematical neuro-oncology: using a simple proliferation and invasion tumor model to inform clinical practice. Bull Math Biol. doi:10.1007/s11538-015-0067-7

Keener J, Sneyd J (2009) Mathematical physiology I: cellular physiology, 2nd edn. Springer, Berlin

Mangel M (2014) Stochastic dynamic programming illuminates the link between environment, physiology, and evolution. Bull Math Biol. doi:10.1007/s11538-014-9973-3

May R, McLean AR (eds) (2007) Theoretical ecology: principles and applications, 3rd edn. Oxford University Press, Oxford

Murray JD (2002) Mathematical biology I: an introduction, 3rd edn. Springer, Berlin

Murray JD (2003) Mathematical biology II: spatial models and biomedical applications, 3rd edn. Springer, Berlin

Othmer HG, Painter K, Umulis D, Xue C (2009) The intersection of theory and application in elucidating pattern formation in developmental biology. Math Model Nat Phenom. 4(4):3-82

Turing MA (1952) The chemical basis of morphogenesis. Philos Trans R Soc Lond B 237:37-72

Tyson JJ, Novak B (2014) Bistability, oscillations and traveling waves in frog egg extracts. Bull Math Biol. doi:10.1007/s11538-014-0009-9

Umulis DM, Othmer HG (2014) The role of mathematical models in understanding pattern formation in developmental biology. Bull Math Biol. doi:10.1007/s11538-014-0019-7 